\title{
Multipartite bound entangled states that violate Bell's inequality
}

\author{
W. Dür \\ Institut für Theoretische Physik, Universität Innsbruck, A-6020 Innsbruck, Austria
}

(October 24, 2018)

\begin{abstract}
We study the relation between distillability of multipartite states and violation of Bell's inequality. We prove that there exist multipartite bound entangled states (i.e. non-separable, non-distillable states) that violate a multipartite Bell inequality. This implies that (i) violation of Bell's inequality is not a sufficient condition for distillability and (ii) some bound entangled states cannot be described by a local hidden variable model.
\end{abstract}

03.65.Ud, 03.67.-a

Since the celebrated work by Bell [1], it is evident that quantum mechanics is not compatible with local realist theories. In fact, Gisin [2] and Gisin and Peres [3] showed that all bipartite entangled pure states violate the BellCHSH inequality 任. This rules out the existence of a local hidden variable (LHV) model which is capable of describing all statistical correlations predicted by quantum mechanics for such states. These results were readily generalized by Popescu and Rohrlich [5] to multipartite entangled pure states of arbitrary dimension.

However, for quantum systems in mixed states, the situation is much more complicated and we still lack a complete classification of mixed states into "local" and "non-local" ones. Although structural knowledge about entanglement has increased rapidly in the last decade, many open questions remain to be answered, in particular concerning the relation of certain entanglement properties to the existence of LHV models.

While it is obvious that separable mixed states [7] can be described by a LHV model, Werner showed in his pioneering paper [6] that also a certain class of entangled mixed states - now called Werner states - do not violate any Bell-type inequality. This was done by explicitly constructing a LHV model which can simulate the results of any single (i.e. nonsequential) measurement performed at each side. However, some years later Popescu [8] realized that also sequential measurements can be considered, and showed that most of the Werner states exhibit violation of local realism if sequences of measurements are taken into account. This so called "hidden nonlocality" is revealed by a sequence of two measurements, where the first measurement is used to select a certain subensemble of pairs - those pairs which produce a specific outcome - while the second measurement tests the Bell observable on the subensemble. If the subensemble does not satisfy Bell's inequality, then one concludes that the initial ensemble violates local realism. In fact, applying a similar reasoning to collective tests of particles [9], it was shown that all inseparable states of two qubits [10] violate local realism. More generally, all distillable 11],12 states violate local realism. It is an open problem whether violation of Bell's inequality which seems to be a rather strong requirement-already implies distillability [13].

While in systems of two qubits all entangled states are distillable [10] (and thus violate local realism), this turned out to be false for higher dimensional systems. The Horodecki 16 discovered states which, although being non-separable and thus entangled, are not distillable. Those states are called bound entangled. The role of bound entangled states is not entirely clear yet. Although they can not be useful for any quantum information processing task directly (since they are non-distillable), it was nevertheless shown that they allow to perform certain processes (e.g. quasidistillation) [17] which cannot be performed using local operations and classical communication alone. In particular, it is not known whether bound entangled states violate local realism or not. This paper is aimed to shine some light on these questions.

We will, however, tackle this problem not in its bipartite setting but rather in the multipartite setting. To this aim, we consider $N$ spatially separated parties. A generalization of the Bell-CHSH inequality to multipartite systems is due to Mermin 18], and was further developed e.g. in Ref. 19] (see also Refs. 20 23] for some recent developments). Also the notion of separability and distillability can be readily generalize to multipartite systems (see e.g. Refs. [24.25]). We have that a $N$-partite state $\rho$ is called (fully) separable iff it can be written as a convex combination of (unnormalized) product states, i.e.

$\rho=\sum_{i}\left|a_{i}\right\rangle_{\text {party } 1}\left\langle a_{i}|\otimes| b_{i}\right\rangle_{\text {party2 }}\left\langle b_{i}|\otimes \ldots \otimes| n_{i}\right\rangle_{\text {party } N}\left\langle n_{i}\right|$.

If $\rho$ is not fully separable, it is entangled. Note that fully separable states are those which can be prepared locally. Due to the fact that many different kinds of multipartite pure state entanglement exist, there are various kinds of distillability [25. We will however not distinguish between those possible kinds of entanglement but rather say that a state $\rho$ is distillable, iff — by means of local operations and classical communication - out of an arbitrary number of identical copies of a state $\rho$ some entangled pure state can be created. If no entangled pure state whatsoever can be created, $\rho$ is non-distillable. As shown in Ref. 24] (see also [26]), there exist also multipartite bound entangled states, i.e. states which are not (fully) separable and hence entangled, but which are non-distillable. Here we investigate multipartite bound 
entangled states and consider the relation of their distillability to the existence of a LHV model. We show that there exist multipartite bound entangled states which violate Bell's inequality. This means on the one hand that violation of Bell's inequality does not imply distillability, and on the other hand that some bound entangled states violate local realism.

In the remainder of this letter, we consider specific $N$-qubit mixed states $\rho_{N}$ acting on a Hilbert space $\mathcal{H}=\left(\mathbb{C}^{2}\right)^{\otimes N}$. We consider $N$ parties, $A_{1}, \ldots, A_{N}$, at different locations, each of them possessing several qubits. The parties possess $M$ identical copies of $\rho_{N}$, where $M$ can be arbitrary large. Thus the state of all qubits is described by the density operator $\rho_{N}^{\otimes M}$. This ensures that the parties can use distillation protocols [12] in order to obtain entangled pure states between some of them. The states $\rho_{N}$ we consider are given by [27]

$$
\rho_{N}=\frac{1}{N+1}\left(|\Psi\rangle\langle\Psi|+\frac{1}{2} \sum_{k=1}^{N}\left(P_{k}+\bar{P}_{k}\right)\right) .
$$

We have that $|\Psi\rangle$ is a $N$-party Greenberger-HorneZeilinger (GHZ) state [28],

$$
|\Psi\rangle=\frac{1}{\sqrt{2}}\left(\left|0^{\otimes N}\right\rangle+e^{i \alpha_{N}}\left|1^{\otimes N}\right\rangle\right),
$$

with $\alpha_{N}$ being an arbitrary phase. We denoted by $P_{k}$ a projector on the state $\left|\phi_{k}\right\rangle$, where $\left|\phi_{k}\right\rangle$ is a product state which is $|1\rangle$ for party $A_{k}$ and $|0\rangle$ for all other parties, i.e. $\left|\phi_{k}\right\rangle=|0\rangle_{A_{1}}|0\rangle_{A_{2}} \ldots|1\rangle_{A_{k}} \ldots|0\rangle_{A_{N-1}}|0\rangle_{A_{N}}$. The projector $\bar{P}_{k}$ is obtained from $P_{k}$ by replacing all zeros by ones and vice versa.

For the states $\rho_{N}(2)$ we show

(i) The states $\rho_{N}$ are bound entangled, i.e. nonseparable and non-distillable if the number of parties $N \geq 4$.

(ii) The states $\rho_{N}$ violate the Mermin-Klyshko inequality if the number of parties $N \geq 8$ and can thus not be described by a LHV model.

We start out by showing (i), i.e. $\rho_{N}$ is bound entangled. One readily verifies that $\rho_{N} T_{A_{k}}$ is a positive operator $\forall k$, where $T_{A_{k}}$ denotes partial transposition with respect to party $A_{k}$ 29. This already implies that $\rho_{N}$ is nondistillable 25. To see this, assume on the opposite that one can distill some bi- or multipartite entangled pure state. As shown below (Lemma 1), one can always create by means of local operations from any multipartite entangled pure state a maximally entangled bipartite pure state shared between two of the parties, say $A_{i}$ and $A_{j}$. Thus the resulting state has non-positive partial transposition with respect to parties $A_{i}$ and $A_{j}$, while the initial state $\rho_{N}$ has positive partial transposition. Due to the fact that by means of local operations and classical communication, one cannot change the positivity of the partial transposition [17], we have the desired contradiction, hence $\rho_{N}$ is not distillable.
On the other hand, $\rho_{N}$ can easily seen to be entangled for $N \geq 4$, e.g. by observing that $\rho_{N}^{T_{A_{k}} A_{l}}$ is not a positive operator for $k \neq l$ and $N \geq 4$. This already implies that $\rho_{N}$ is not fully separable, as positivity of all possible partial transpositions is a necessary condition for (full) separability in multipartite systems 30,22]. It remains to show the announced

Lemma 1: Given a single copy of an $N$-party entangled pure state $|\Phi\rangle$ of arbitrary dimension, one can always create with non-zero probability of success by means of local operations a maximally entangled bipartite pure state shared among some of the parties.

Proof: One may write $|\Phi\rangle$ in its Schmidt decomposition with respect to any party $A_{k}$. Since $|\Phi\rangle$ is entangled, there exist at least one party, say $A_{1}$, where one obtains a minimal number of two non-zero Schmidt coefficients (otherwise $|\Phi\rangle$ would be a $N$-party product state). That is,

$$
|\Phi\rangle=\sum_{k=0}^{d} \lambda_{k}|k\rangle_{A_{1}}\left|\varphi_{k}\right\rangle_{A_{2} \ldots A_{N}}
$$

where $\left\langle k \mid k^{\prime}\right\rangle=\left\langle\varphi_{k} \mid \varphi_{k^{\prime}}\right\rangle=\delta_{k k^{\prime}}$ and $d \geq 2$. For simplicity, let us assume that $d=2$ and $\lambda_{0}=\lambda_{1}=1 / \sqrt{2}$ (this can always be accomplished by a filtering measurement in $A_{1}$, e.g. using $\left.O_{A_{1}}=\lambda_{1}|0\rangle\left\langle 0\left|+\lambda_{0}\right| 1\right\rangle\langle 1|\right)$. Now, either (a) both $\left|\varphi_{0}\right\rangle$ and $\left|\varphi_{1}\right\rangle$ are product states, or (b) at least one of them, say $\left|\varphi_{0}\right\rangle$, is entangled.

In case of (a), $\left|\varphi_{0}\right\rangle$ and $\left|\varphi_{1}\right\rangle$ have to be locally orthogonal in at least one location, say at $A_{2}$ (this is due to the fact that $\left\langle\varphi_{0} \mid \varphi_{1}\right\rangle=0$ ), i.e. $\left|\varphi_{0}\right\rangle=|0\rangle_{A_{2}}\left|\chi_{3}\right\rangle_{A_{3}} \ldots\left|\chi_{N}\right\rangle_{A_{N}}$ and $\left|\varphi_{1}\right\rangle=|1\rangle_{A_{2}}\left|\tilde{\chi}_{3}\right\rangle_{A_{3}} \ldots\left|\tilde{\chi}_{N}\right\rangle_{A_{N}}$. There may exist $l$ locations $A_{k}, l \leq N-2$ for which $\left|\chi_{k}\right\rangle=\left|\tilde{\chi}_{k}\right\rangle$. Each of the other parties $A_{k}$ can apply a local filtering measurement of the form $O_{A_{k}}=|0\rangle\left\langle\chi_{k}^{\prime}|+| 1\right\rangle\left\langle\tilde{\chi}_{k}^{\prime}\right|$, where $\left\{\left|\chi_{k}^{\prime}\right\rangle,\left|\tilde{\chi}_{k}^{\prime}\right\rangle\right\}$ is the biorthonormal basis to $\left\{\left|\chi_{k}\right\rangle,\left|\tilde{\chi}_{k}\right\rangle\right\}$, i.e. $\left\langle\tilde{\chi}_{k} \mid \chi_{k}^{\prime}\right\rangle=\left\langle\chi_{k} \mid \tilde{\chi}_{k}^{\prime}\right\rangle=0$ and $\left\langle\tilde{\chi}_{k} \mid \tilde{\chi}_{k}^{\prime}\right\rangle=\left\langle\chi_{k} \mid \chi_{k}^{\prime}\right\rangle=1$. One readily observes that this leads to the creation of a $N-l$ party GHZ state (3), from which - by means of local measurements in the basis $\{|+\rangle,|-\rangle\}$, where $| \pm\rangle=1 / \sqrt{2}(|0\rangle \pm|1\rangle)$ at the remaining locations-a maximally entangled bipartite pure state shared between any two out of the remaining $N-l$ parties can be created deterministically.

In case of (b), one measures in $A_{1}$ the projector $P_{A_{1}}=$ $|0\rangle\langle 0|$ and is left with an entangled state of $N-1$ (or less) particles. This situation is similar to the one we started with, however the number of entangled systems decreased. One proceeds in the same vain with the remaining systems until (a) applies, which happens in the worst case if only two entangled particles are left. Finally, one obtains at least a maximally entangled bipartite state shared among two of the parties which concludes the proof of the lemma.

Note that from Lemma 1 follows the non-existence of a LHV model for all multipartite entangled pure states of arbitrary dimension which describes properly also sequences of measurements (see Ref. [5] for the stronger 
result of inconsistency with local realism even for single measurements per site). Following the reasoning of Popescu [8 (see also [31]) (adopted to the multipartite case), the violation of Bell's inequality of a subensembles of states (obtained e.g. by local filtering measurements) ensures that also the original ensemble violates local realism. Since, according to Lemma 1, from any multipartite entangled pure state a maximally entangled bipartite pure state can be created - which clearly maximally violates Bell's inequality - the claim follows.

We now turn to (ii) and show that $\rho_{N}$ violates the Mermin-Klyshko inequality for $N \geq 8$. Let $a_{j}, a_{j}^{\prime}$ be two vectors on the unit sphere which indicate two possible measurement directions for party $A_{j}$. The corresponding observables are given by $O_{j}=\sigma_{a_{j}}, O_{j}^{\prime}=\sigma_{a_{j}^{\prime}}$. Up to a normalization factor, any $k$-qubit Bell inequality involving two observables per qubit can be written as

$$
\left|\left\langle\mathcal{B}_{k}\right\rangle\right| \leq 1,
$$

where $\mathcal{B}_{k} \equiv \mathcal{B}_{k}\left(a_{1}, a_{2}, \ldots, a_{k}, a_{1}^{\prime}, a_{2}^{\prime}, \ldots, a_{k}^{\prime}\right)$ is the corresponding Bell operator. We consider the Mermin-Kyshko inequalities [18,19] for $N$ qubits, whose corresponding Bell operator is defined recursively as 23

$$
\mathcal{B}_{k}=\frac{1}{2} \mathcal{B}_{k-1} \otimes\left(\sigma_{a_{k}}+\sigma_{a_{k}^{\prime}}\right)+\frac{1}{2} \mathcal{B}^{\prime}{ }_{k-1} \otimes\left(\sigma_{a_{k}}-\sigma_{a_{k}^{\prime}}\right),
$$

where $\mathcal{B}^{\prime}{ }_{k}$ is obtained from $\mathcal{B}_{k}$ by exchanging all the $a_{k}$ and $a_{k}^{\prime}$.

We choose the same measurement directions in all $N$ locations, $\sigma_{a_{j}}=\sigma_{x}$ and $\sigma_{a_{j}^{\prime}}=\sigma_{y} \forall j$, where $\sigma_{x}, \sigma_{y}$ are Pauli matrices. One readily verifies that in this case $\mathcal{B}_{N}$ can be written as

$$
\mathcal{B}_{N}=2^{\frac{N-1}{2}}\left(e^{i \beta_{N}}\left|1^{\otimes N}\right\rangle\left\langle 0^{\otimes N}\left|+e^{-i \beta_{N}}\right| 0^{\otimes N}\right\rangle\left\langle 1^{\otimes N}\right|\right),
$$

with $\beta_{N} \equiv \pi / 4(N-1)$. Using that $\operatorname{tr}\left(\mathcal{B}_{N} P_{k}\right)=$ $\operatorname{tr}\left(\mathcal{B}_{N} \bar{P}_{k}\right)=0 \forall k$ and $\operatorname{tr}\left(\mathcal{B}_{N}|\Psi\rangle\langle\Psi|\right)=2^{\frac{N-1}{2}}$ when fixing the phase $\alpha_{N}=\beta_{N}$ in $|\Psi\rangle$ (3), we have

$$
\operatorname{tr}\left(\mathcal{B}_{N} \rho_{N}\right)=\frac{1}{N+1} 2^{\frac{N-1}{2}},
$$

which fulfills $\operatorname{tr}\left(\mathcal{B}_{N} \rho_{N}\right)>1$ iff $N \geq 8$. Thus the states $\rho_{N}$ (2) with the choice $\alpha_{N}=\pi / 4(N-1)$ violate the Mermin-Klyshko inequality for $N \geq 8$ as announced.

To conclude, we have shown that certain multipartite bound entangled states violate a multipartite Bell inequality. This implies that (i) violation of Bell's inequality is not a sufficient condition for distillability and (ii) there does not exist a local hidden variable model for certain bound entangled states. Note that the states $\rho_{N}$ for sufficiently large $N$ violate the Mermin-Klyshko inequality directly, and no sequence of measurements, eventually performed on a tensor product of the states, is required to rule out the existence of a LHV model as in the case of "hidden nonlocality". There remain a number of open problems concerning the relation of inseparability to the existance of a LHV model [32]. In particular, it is not known whether all bipartite bound entangled states, those with positive partial transposition as well as the conjectured ones with non-positive partial transposition [33], can be described by a local hidden variable model or not.

I thank G. Vidal for an essential contribution in the proof of Lemma 1, and J. I. Cirac and G. Giedke for interesting discussions. This work was supported by the Austrian SF under the SFB "control and measurement of coherent quantum systems" (Project 11), the European Community under the TMR network ERBFMRX-CT96-0087 and project EQUIP (contract IST1999-11053), the ESF, and the Institute for Quantum Information GmbH.

[1] J. S. Bell, Physics 1, 195 (1964)

[2] N. Gisin, Phys. Lett. A 154, 201 (1991)

[3] N. Gisin and A. Peres, Phys. Lett. A. 162, 15 (1992).

[4] J. F. Clauser, M. A. Horne, A. Shimony and R. A. Holt, Phys. Rev. Lett. 23, 880 (1969).

[5] S. Popescu and D. Rohrlich, Phys. Lett. A. 166, 293 (1992).

[6] R. F. Werner, Phys. Rev. A 40, 4277 (1989).

[7] A bipartite mixed state of two parties $A$ and $B$ is said to be separable iff it can be written as a convex combination of product states, $\rho=\sum p_{i}\left|a_{i}\right\rangle_{A}\left\langle a_{i}|\otimes| b_{i}\right\rangle_{B}\left\langle b_{i}\right|$. Otherwise, $\rho$ is entangled.

[8] S. Popescu, Phys. Rev. Lett. 74, 2619 (1995).

[9] A. Peres, Phys. Rev. A 54, 2685 (1996)

[10] M. Horodecki, P. Horodecki and R. Horodecki, Phys. Rev. Lett. 78, 574 (1997)

[11] A bipartite state $\rho$ is called distillable, iff — by means of local operations and classical communication- out of an arbitrary number of identical copies of the state a maximally entangled bipartite pure state, e.g. $\left|\Phi^{+}\right\rangle=$ $1 / \sqrt{2}(|00\rangle+|11\rangle)$, can be created.

[12] C. H. Bennett, G. Brassard, S. Popescu, B. Schumacher, J. A. Smolin and W. K. Wootters, Phys. Rev. Lett. 76, 722 (1996); C. H. Bennett, H. J. Bernstein, S. Popescu and B. Schumacher, Phys. Rev. A 53, 2046 (1996).

[13] This seems to be the intuition of Peres, who conjectures in Ref. 14. that all states with positive partial transposition can be described by a LHV model. Results in favour of this conjecture were recently obtained in Ref. [15].

[14] A. Peres, Found. Phys. 29, 589 (1999)

[15] R. F. Werner and M. M. Wolf, Phys. Rev. A 61, 062102 (2000).

[16] M. Horodecki, P. Horodecki and R. Horodecki, Phys. Rev. Lett. 80, 5239 (1998).

[17] P. Horodecki, M. Horodecki and R. Horodecki, Phys. Rev. Lett. 82, 1056 (1999).

[18] N. D. Mermin, Phys. Rev. Lett. 65, 1838 (1990).

[19] A. V. Belinskii and D. N. Klyshko, Phys. Usp. 36, 653 
(1993).

[20] N. Gisin and H. Bechmann-Pasquinucci, Phys. lett. A 246, 1 (1998)

[21] R. F. Werner and M .M. Wolf, quant-ph/0102024 (2001).

[22] M. Zukowski and C. Brukner, quant-ph/0102039 (2001).

[23] V. Scarani and N. Gisin, quant-ph/0103068 (2001).

[24] W. Dür, J. I. Cirac, and R. Tarrach, Phys. Rev. Lett. 83, 3562 (1999); W. Dür and J. I. Cirac, Phys. Rev. A 61, 042314 (2000). W. Dür and J. I. Cirac, quant-ph 0011025;

[25] W. Dür and J. I. Cirac, Phys. Rev. A 62, 022302 (2000).

[26] J. Smolin, quant-ph/0001001

[27] Note that $\rho_{N}$ belongs - up to an irrelevant phase factorto a family of $N$-partite mixed states studied in detail in Refs. 24,25. The corresponding parameters are given by $\lambda_{0}^{+}=1 /(N+1), \lambda_{0}^{-}=0$ and $\lambda_{k}=1 /(2 N+2)$ if $k$ corresponds to a bipartite splitting one party - rest and $\lambda_{k}=0$ otherwise.

[28] D. M. Greenberger, M. Horne, A. Zeilinger, Bell's theorem, Quantum Theory, and Conceptions of the Universe, ed. M. Kafatos, Kluwer, Dordrecht 69 (1989);

[29] For a definition of the partial transposition in bipartite and multipartite systems see 30,24.

[30] A. Peres, Phys. Rev. Lett. 77, 1413 (1996).

[31] M. Zukowski, R. Horodecki, M. Horodecki and P. Horodecki, Phys. Rev. A 58, 1694 (1998).

[32] B. M. Terhal, Phys. Lett. A 271, 319 (2000)

[33] W. Dür, J. I. Cirac, M. Lewenstein and D. Bruß, Phys. Rev. A 61, 062313 (2000); D. P. DiVincenzo, P. W. Shor, J. A. Smolin, B. M. Terhal and A. V. Thapliyal, Phys. Rev. A 61, 062312 (2000) 